\title{
Migraine History and Breast Cancer Risk: A Systematic Review and Meta-Analysis
}

\author{
Shahab Rezaeian1, Yousef Veisani2 ${ }^{*}$, Mohammad Ghorbani' ${ }^{3}$, Ali Delpisheh4, \\ Hedayat Abbastabar ${ }^{3}$ \\ ${ }^{1}$ Health Promotion Research Center, Zahedan University of Medical Sciences, Zahedan, Iran \\ ${ }^{2}$ Student Research Committee, Ilam University of Medical Sciences, Ilam, Iran \\ ${ }^{3}$ Department of Epidemiology, School of Health, Shiraz University of Medical Sciences, Shiraz, Iran \\ ${ }^{4}$ Department of Clinical Epidemiology, Ilam University of Medical Sciences, Ilam, Iran \\ Email: shahab.rezayan@gmail.com, \\ alidelpisheh@yahoo.com, hedayat.abastabar@yahoo.com
}

Received 24 March 2015; accepted 30 June 2015; published 3 July 2015

Copyright (C) 2015 by authors and Scientific Research Publishing Inc.

This work is licensed under the Creative Commons Attribution International License (CC BY).

http://creativecommons.org/licenses/by/4.0/

\section{(c) (i) Open Access}

\begin{abstract}
Objective: The relationship between migraine and breast cancer risk has been reported inconsistently across different epidemiological studies. This meta-analysis was performed to explore the overall effect of migraine on breast cancer risk. Method: An electronic search of different major databases was conducted, including PubMed, Scopus, ScienceDirect, and the Cochrane library until February $1^{\text {st }}, 2015$. Of 652 retrieved studies, six population-based studies including two cohort studies with 130,812 and four case-control studies with 14,396 people were included in the analysis. Results: There was an inverse relationship between migraine and breast cancer risk (OR = 0.77; 95\% CI: 0.64, 0.92). Conclusion: The results of this meta-analysis showed that women with migraine history have a decreased risk of breast cancer. Further biological studies are needed to address the association.
\end{abstract}

\section{Keywords}

Migraine, Breast cancer, Meta-Analysis

\section{Introduction}

Gender and age-dependent prevalence of migraine was estimated by population based studies [1]-[2]. The prevalence rate of migraine varies in the general population and is more common in women [1] [3].

Breast cancer is the most common cancer as well as is the leading cause of cancer related mortality among "Corresponding author.

How to cite this paper: Rezaeian, S., Veisani, Y., Ghorbani, M., Delpisheh, A. and Abbastabar, H. (2015) Migraine History and Breast Cancer Risk: A Systematic Review and Meta-Analysis. Advances in Breast Cancer Research, 4, 63-70. 
woman worldwide [4]. Nevertheless, the etiology of breast cancer, as a momentous of public health problem, is not well known. Several studies have indicated that different risk factors including genetic, environmental, hormonal, lifestyle and physiological factors have an influence on the development of this malignancy [5]-[6].

Over the past decade numerous systematic review and meta-analysis studies were conducted to examine of an association between migraine and other diseases such as cardiovascular disease [7], ischemic stroke risk [8], and mortality [9]. On the other hand, there were also several population based studies which had extensively evaluated the relationship between migraine and female cancer, but with conflicting results. Some studies had reported that women with a history of migraine are at lower risk of breast cancer [10]-[14], whereas others reported no association [15]. In addition, no significant association was found between migraine history and risk of endometrial cancer [16].

We, therefore, aimed to explore the relationship between migraine history and breast cancer risk and to quantify the risk by conducting a meta-analysis. We also aimed to evaluate whether the associations varied by age at migraine diagnosis, histological subtype and hormone receptor status of breast cancer.

\section{Methods}

\subsection{Search Strategies}

We conducted a systematic search of all studies designed cohort and case-control studies addressing the association between migraine and breast cancer. A comprehensive literature search of numerous electronic databases including PubMed, Scopus, ScienceDirect and the Cochrane library was done up until February $1^{\text {st }}, 2015$.

A search strategy was conducted using the following search terms in the titles, abstract or key words: migraine, migraine disorder, breast, breast cancer, case-control studies, cohort studies, and observational studies. The citations and references listed in retrieved articles were also reviewed to identify additional related studies.

\subsection{Inclusion Criteria}

Two authors reviewed the retrieved studies independently to identify eligible studies by using the following criteria: 1) Study design: cohort or case-control study, 2) the breast cancer patients were confirmed by medical records 3) the migraine status was confirmed by medical records, 4) the numbers of case and control (in case-control studies) and exposed and non-exposed groups (in cohort studies) were reported or the relevant data was available to calculate the odds ratio (OR) or risk ratio(RR). Women with breast cancer were included regardless of age, race, menopausal and marital status.

\subsection{Data Extraction and Quality Assessment}

Two independent authors read full-text articles and the following information was extracted: name of the first author, publication year and location of study conduction, study design (cohort or case-control study), number of cases and controls or exposed and non-exposed, age at migraine diagnosis, histological subtype (ductal and lobular), and hormone (estrogen (ER) and progesterone (PR)) receptor status (ER+/PR+, ER+/PR-, ER-/PR-). The quality of studies was assessed according to the STROBE statement and the studies according with STROBE criteria were defined as high-quality studies [17].

\subsection{Statistical Analysis}

The pooled ORs with 95\% confidence interval (CI) estimating the association between migraine and breast cancer were obtained using the random effect model. Cochran's Q test was used to identify the heterogeneity of results across studies and it was quantified using the $\mathrm{I}^{2}$ statistic. Q statistic with p-value $<0.10$ or $\mathrm{I}^{2}$ statistic $>50 \%$ was considered as significant heterogeneity across studies. The between-study variance was estimated using tau-squared $\left(\mathrm{t}^{2}\right.$ or Tau $\left.{ }^{2}\right)$ statistic [18]. We used Egger's linear regression test [19] to investigate publication bias ( $p<0.05$ set as significant level). Meta-analysis was performed by the comprehensive meta-analysis software version 2.0. The PRISMA statement was used as a guide in the reporting of this study [20].

\section{Results}

\subsection{Description of Studies}

Table 1 presents the characteristics of the studies included in the meta-analysis. Of 652 retrieved studies, we 
Table 1. Characteristics of the studies included in the meta-analysis.

\begin{tabular}{cccccccc}
\hline & Data source & Study type & Sampling & Cases & Control & Exposed & Non-exposed \\
\hline Ghorbani et al. $2015[14]$ & CR & Case-control & & 347 & 300 & - & - \\
Lowry et al. $2014[12]$ & SEER & Case-control & Population-based & 715 & 376 & - & - \\
Li et al. $2009[11]$ & SEER & Case-control & Population-based & 4568 & 4678 & - & - \\
Mathes et al. $2008[13]$ & CSS & Case-control & Population-based & 1938 & 1474 & - & - \\
Li et al. $2010[10]$ & WHI & Cohort & Population-based & - & - & 10464 & 80652 \\
Winter et al. $2013[15]$ & WHS & Cohort & Population-based & - & - & 7318 & 32378 \\
\hline
\end{tabular}

CR: Cancer Registry at the oncology center of Isfahan University of Medical Sciences, Iran; SEER: Surveillance Epidemiology and End Results cancer registry; CSS: Cancer Surveillance System; WHI: Women’s Health Initiative; WHS: Women’s Health Study.

eventually included 6 population-based studies in the meta-analysis including two cohort and four case-control studies involving 130812 and 14396 people, respectively. Figure 1 presents the flowchart of the literature search process.

\subsection{Summary and Subgroup Analyzed Results}

Table 2 illustrates the summary effect sizes of breast cancer risk and migraine history by age at migraine diagnosis, histological subtype and hormone receptor status.

\subsection{Association of Migraine History and Breast Cancer Risk}

Summary results of six population-based studies (Figure 2) indicated that having any history of migraine was inversely associated with breast cancer risk (OR $=0.77$; 95\% CI: 0.64, 0.92).

\subsection{Association of Age at Migraine Diagnosis and Breast Cancer Risk}

We stratified the migraine history by the age at diagnosis (Figure 3). Pooled result from three studies showed that both age at migraine diagnosis $<20$ years and over 20 years decreased the risk of breast cancer $(\mathrm{OR}=0.66$, $95 \%$ CI 0.48, 0.90 and OR $=0.68$, 95\% CI 0.47, 0.99, respectively). That means the women who reported a clinical diagnosis of migraine in age $<20$ or $>20$ years were significantly at lower risk of breast cancer.

\subsection{Association of Migraine History and Breast Cancer Risk by Histological Subtype}

The summary results of associations between migraine history and breast cancer risk showed that the association does not vary by histological type compared to non-migraineur women (Figure 4). That means women with a history of migraine had similarly reduced risks of both ductal and lobular carcinomas (OR $=0.84$, 95\% CI 0.70 1.01 and OR $=0.79$, 95\% CI 0.70, 0.90, respectively). Test for histological subgroup differences was not significant $(\mathrm{p}=0.585)$. Overall, women with migraine history had a $19 \%$ reduced risk of breast cancer regardless of histological subgroup.

\subsection{Association of Migraine History and Breast Cancer Risk by Hormone Receptor Status}

Pooled result showed that women with migraine history had a 15\% reduced risk of breast cancer regardless of hormone receptor status (OR $=0.85,95 \%$ CI 0.77, 0.94). Figure 5 shows the forest plot of OR estimates of association between migraine and breast cancer risk by hormone receptor status. We did not observe significant differences with regard to hormone receptor status $(\mathrm{p}=0.253)$. On the other hand, migraineur women had a lower risk of $\mathrm{ER}+\mathrm{PR}+(\mathrm{OR}=0.80,95 \% \mathrm{CI} 0.68,0.93), \mathrm{ER}+\mathrm{PR}-(\mathrm{OR}=0.82,95 \% \mathrm{CI} 0.67,0.99)$, and $\mathrm{ER}-/ \mathrm{PR}$ - breast cancer $(\mathrm{OR}=0.97,95 \% \mathrm{CI} 0.80,1.17)$.

\subsection{Heterogeneity and Publication Bias}

Significant heterogeneity between studies was noted in the studies. The results of Q-test showed that the studies 


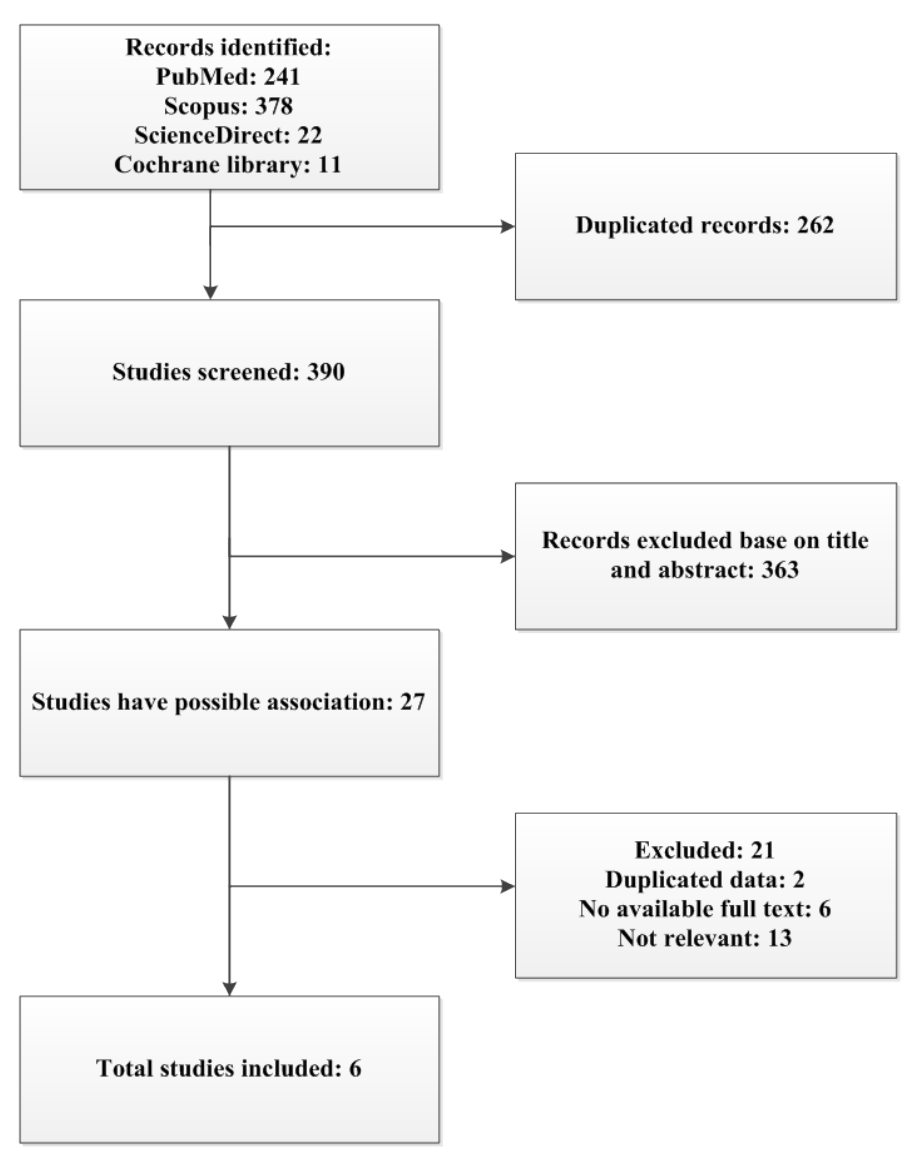

Figure 1. Flowchart of the literature search in the systematic review and meta-analysis.

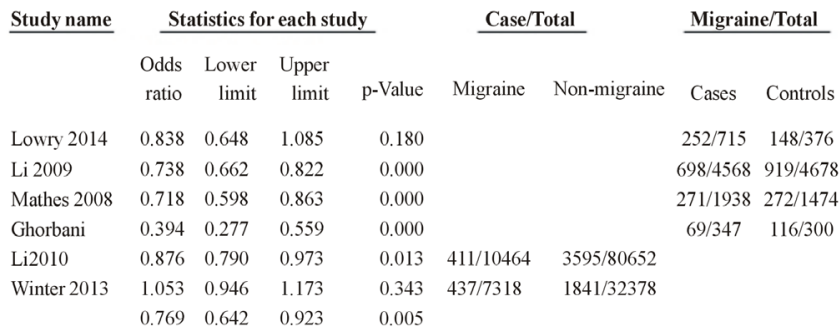

Heterogeneity: $\mathrm{Q}$-value $=43.8, \mathrm{df}=\mathbf{5}(\mathrm{p}=0.001) ; \mathrm{I}^{2}=88.5 \% ; \mathrm{Tau}^{2}=0.04$

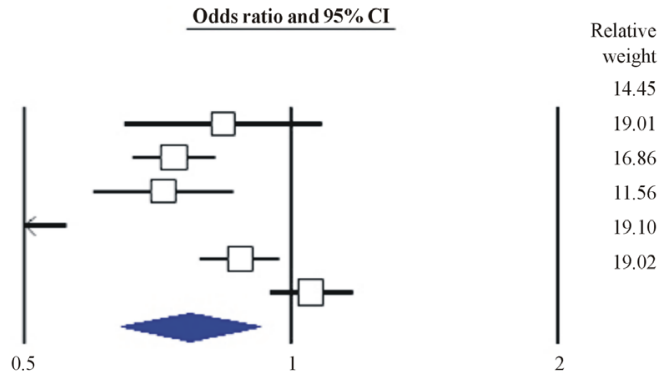

lative

4.45

19.01

Figure 2. Forest plot of odds ratio estimate of association between migraine and breast cancer risk in the all studies.

were significantly heterogeneous $(\mathrm{p}<0.001)$. The $\mathrm{I}^{2}$ and tau statistics were $88.5 \%$ and 0.04 , respectively (Figure 2). Of six included studies assessed the effect of migraine on breast cancer risk, five studies reported negative associations (four out of which were statistically significant) and one study reported non-significant positive association. The heterogeneity between studies with related statistics is shown in Table 2. We couldn't assess the publication bias using the funnel plot, because the number of included studies in the meta-analysis was relatively small. No publication bias was detected by Egger's regression ( $p=0.647)$.

\section{Discussion}

The results of this meta-analysis revealed a significant negative relationship between migraine history and risk of breast cancer, women with migraine history have a 23\% reduced risk of breast cancer. There was evidence of significant heterogeneity in the meta-analysis. We used the Q-test to detect of heterogeneity. Accordingly, this 


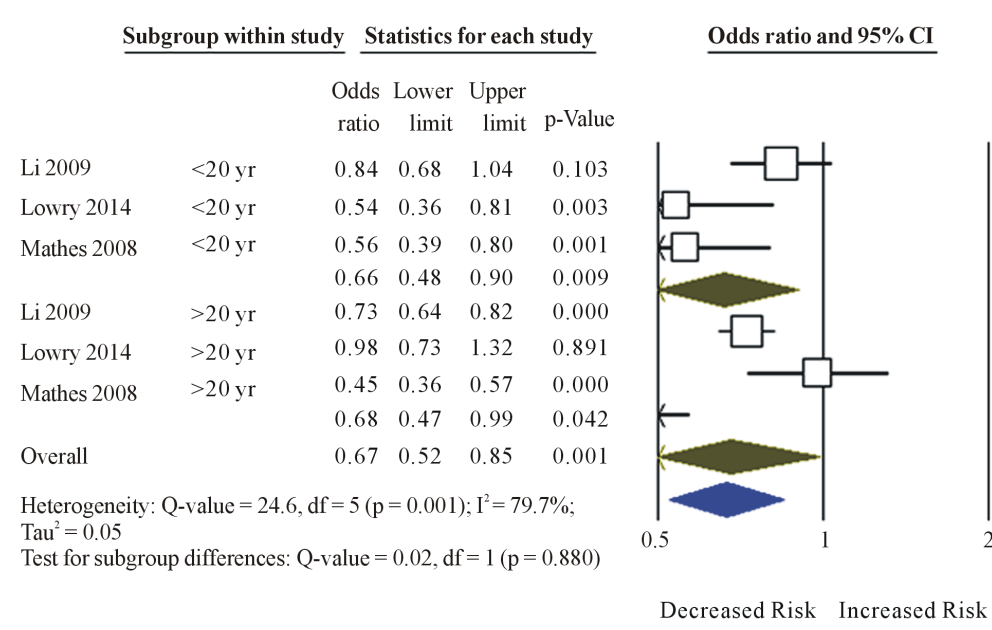

Figure 3. Forest plot of odds ratio estimates of breast cancer risk by age at migraine diagnosis.

$\underline{\text { Subgroup within study }}$ Statistics for each study

Odds ratio and $95 \% \mathrm{CI}$

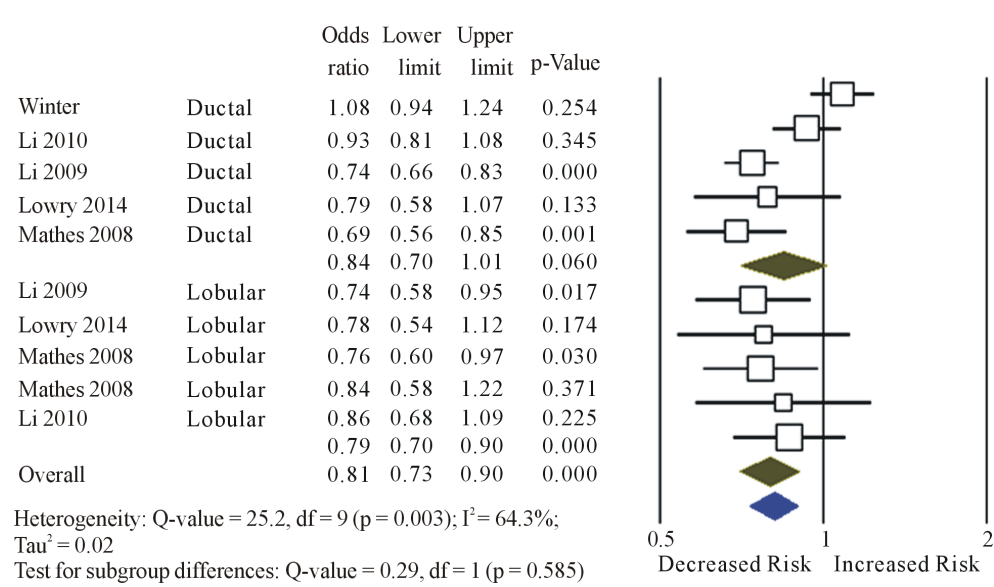

Figure 4. Forest plot of odds ratio estimates of association between migraine and breast cancer risk by histological subtype.

Table 2. Summary effect sizes of breast cancer risk and migraine status by age at migraine diagnosis, histological subtype and hormone receptor status.

\begin{tabular}{cccccccccc}
\hline \multirow{2}{*}{ Subgroup } & OR & \multicolumn{2}{c}{$95 \%$ CI } & p value & No. of studies & \multicolumn{4}{c}{ Heterogeneity } \\
\cline { 7 - 10 } & & & & & Q-value & P for Q test & I-squared & Tau squared \\
\hline$<20 \mathrm{yr}$ & 0.66 & 0.48 & 0.90 & 0.009 & 3 & 5.88 & 0.053 & 66.0 & 0.05 \\
$>20 \mathrm{yr}$ & 0.68 & 0.47 & 0.99 & 0.042 & 3 & 18.61 & 0.001 & 89.3 & 0.09 \\
ER-/PR- & 0.97 & 0.80 & 1.17 & 0.754 & 4 & 5.61 & 0.132 & 46.5 & 0.02 \\
ER+/PR- & 0.82 & 0.67 & 0.99 & 0.043 & 4 & 3.78 & 0.287 & 20.6 & 0.01 \\
ER+/PR+ & 0.80 & 0.68 & 0.93 & 0.003 & 4 & 10.23 & 0.017 & 70.7 & 0.02 \\
Ductal & 0.84 & 0.70 & 1.01 & 0.060 & 5 & 23.14 & 0.001 & 82.7 & 0.03 \\
Lobular & 0.79 & 0.70 & 0.90 & 0.001 & 5 & 0.99 & 0.912 & 0 & 0 \\
\hline
\end{tabular}

Age at migraine diagnosis: $<20$ and $>20$ years; Histological subtype: ductal and lobular; Hormone receptor status: estrogen and progesterone receptor $(\mathrm{ER}+/ \mathrm{PR}+, \mathrm{ER}+/ \mathrm{PR}-$ and $\mathrm{ER}-/ \mathrm{PR}-)$. 


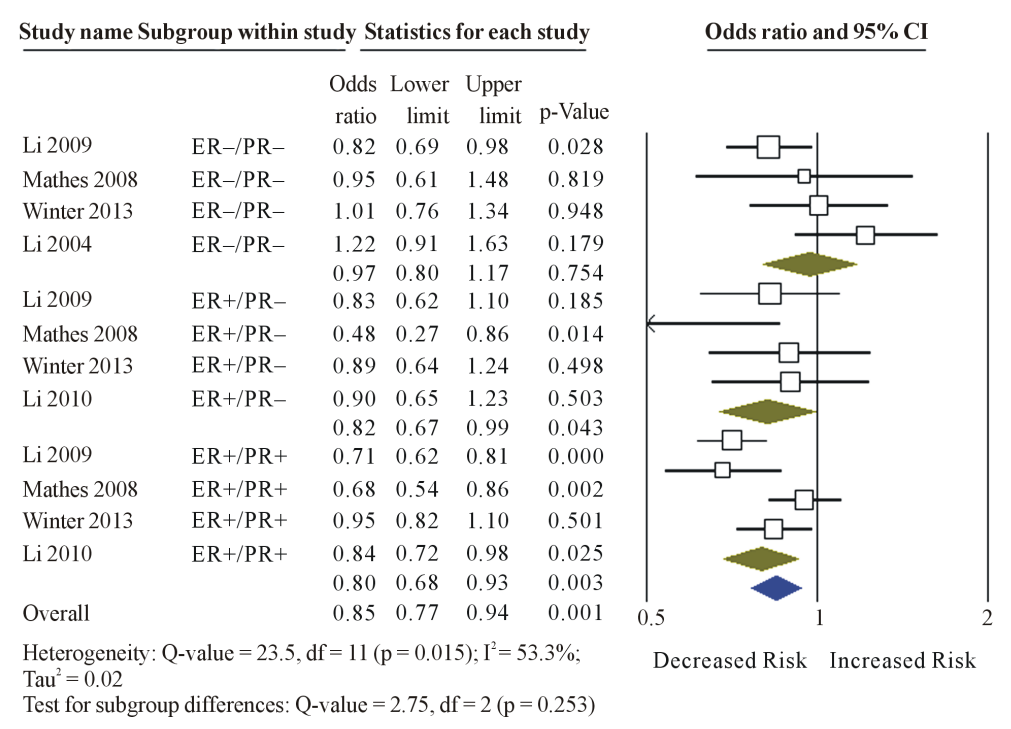

Figure 5. Forest plot of odds ratio estimates of association between migraine and breast cancer risk by hormone receptor status.

issue should be noted that the Q-test has low power when the included studies are small [18]. Therefore, some parts of the observed heterogeneity in the results can be attributed to the number of studies (including six studies) included in the meta-analysis. Subgroup meta-analyses by age at migraine diagnosis, histological subtype and hormone receptor status of breast cancer were also conducted (Table 2). Five included studies considered both histological subtype (including ductal and lobular) and hormone receptor status (including ER+/PR+, ER+/PR-, ER-/PR-) of breast cancer, and only three studies considered age at migraine diagnosis [11]-[13].

With regard to the subgroup analysis, the pooled results illustrate a significant correlation between migraine history and decreased risk of breast cancer (Figures 3-5). The summary estimate from the ER+/PR+ was more protective than in $\mathrm{ER}+\mathrm{PR}-$ or $\mathrm{ER}-/ \mathrm{PR}-(0.80$ vs. 0.82 and 0.97 , respectively). For histological subtype, the summary estimate was also more protective for lobular than for ductal carcinomas ( 0.79 vs. 0.84$)$. Biologically, both migraine and breast cancer are related to estrogen hormone [10]-[11] [15], but the precise biology and hormonal pathways of migraine relevant to a potential reduction in breast cancer risk are poorly understood [13]. Prior studies showed an independent association between migraine and ER- breast cancer but reported a lower risk of ER+ [15]. Some studies mentioned this hypothesizes that migraine may be particularly associated with a reduced risk of hormone receptor positive tumors [21]. This association may be confounded by a factor entitled obesity. Previous epidemiological studies reported that obesity may be more strongly associated with ER+ postmenopausal breast cancer [22]-[26].

We stratified the migraine history by age at migraine diagnosis ( $<20$ and $>20$ years). There was no significant difference between migraine and breast cancer by this variable suggesting that age is not strong confounder. One possible explanation for a lower risk of breast cancer in women with a history of migraine could be that such migraineur women are more likely to avoid migraine risk factors such as stress, cigarette smoking and alcohol. The findings of previous study were consistent with this hypothesis, in that alcohol and smoking were less common among migraineurs [27]. Another explanation about this lower risk may relate to specific migraine treatments [12].

Toward our understanding, this study represents the first systematic review of the relationship between migraine history and breast cancer risk. Other strengths of our study were subgroup analysis and all enrolled subject in primary studies was diagnosed based on clinical criteria for migraine and finally large overall sample size.

Following limitations of our study need to be considered. First, three of the included studies were case-control design and potential to recall bias. Second, because of lack of migraine classification according to clinical criteria in the enrolled studies, we could not perform the subgroup analysis based on this variable. Third, heterogeneity was significant and we could not specify source of heterogeneity in our study because of the limited number of available studies. 
In conclusion, the results of this meta-analysis showed that women with migraine history have a decreased risk of breast cancer. Further biological studies are needed to address the association.

\section{Acknowledgements}

This research has not received any specific fund. The authors declare that there is no conflict of interest.

\section{References}

[1] Fernández-de-Las-Peñas, C., Palacios-Ceña, D., Salom-Moreno, J., López-de-Andres, A., Hernández-Barrera, V., Jiménez-Trujillo, I., et al. (2014) Has the Prevalence of Migraine Changed over the Last Decade (2003-2012)? A Spanish Population-Based Survey. PLoS ONE, 9, e110530. http://dx.doi.org/10.1371/journal.pone.0110530

[2] Smitherman, T.A., Burch, R., Sheikh, H. and Loder, E. (2013) The Prevalence, Impact, and Treatment of Migraine and Severe Headaches in the United States: A Review of Statistics from National Surveillance Studies. Headache: The Journal of Head and Face Pain, 53, 427-436. http://dx.doi.org/10.1111/head.12074

[3] Ramage-Morin, P.L. and Gilmour, H. (2014) Prevalence of Migraine in the Canadian Household Population. Health Reports, 25, 10-16.

[4] Siegel, R., Ma, J., Zou, Z. and Jemal, A. (2014) Cancer Statistics. CA: A Cancer Journal for Clinicians, 64, 9-29. http://dx.doi.org/10.3322/caac.21208

[5] WHO (2006) Guidelines for Management of Breast Cancer. Egypt.

[6] McPherson, K., Steel, C.M. and Dixon, J.M. (2000) ABC of Breast Diseases. Breast Cancer-Epidemiology, Risk Factors, and Genetics. British Medical Journal, 321, 624-628. http://dx.doi.org/10.1136/bmj.321.7261.624

[7] Rist, P.M., Diener, H.C., Kurth, T. and Schürks, M. (2011) Migraine, Migraine Aura, and Cervical Artery Dissection: A Systematic Review and Meta-Analysis. Cephalalgia, 31, 886-896. http://dx.doi.org/10.1177/0333102411401634

[8] Etminan, M., Takkouche, B., Isorna, F.C. and Samii, A. (2005) Risk of Ischaemic Stroke in People with Migraine: Systematic Review and Meta-Analysis of Observational Studies. British Medical Journal, 330, 63.

http://dx.doi.org/10.1136/bmj.38302.504063.8F

[9] Schürks, M., Rist, P.M., Shapiro, R.E. and Kurth, T. (2011) Migraine and Mortality: A Systematic Review and Meta-Analysis. Cephalalgia, 31, 1301-1314. http://dx.doi.org/10.1177/0333102411415879

[10] Li, C.I., Mathes, R.W., Bluhm, E.C., Caan, B., Cavanagh, M.F., Chlebowski, R.T., et al. (2010) Migraine History and Breast Cancer Risk among Postmenopausal Women. Journal of Clinical Oncology, 28, 1005-1010. http://dx.doi.org/10.1200/JCO.2009.25.0423

[11] Li, C.I., Mathes, R.W., Malone, K.E., Daling, J.R., Bernstein, L., Marchbanks, P.A., et al. (2009) Relationship between Migraine History and Breast Cancer Risk among Premenopausal and Postmenopausal Women. Cancer Epidemiology, Biomarkers \& Prevention, 18, 2030-2034. http://dx.doi.org/10.1158/1055-9965.EPI-09-0291

[12] Lowry, S.J., Malone, K.E., Cushing-Haugen, K.L. and Li, C.I. (2014) The Risk of Breast Cancer Associated with Specific Patterns of Migraine History. Cancer Causes \& Control, 25, 1707-1715. http://dx.doi.org/10.1007/s10552-014-0479-y

[13] Mathes, R.W., Malone, K.E., Daling, J.R., Davis, S., Lucas, S.M., Porter, P.L., et al. (2008) Migraine in Post-Menopausal Women and the Risk of Invasive Breast Cancer. Cancer Epidemiology, Biomarkers \& Prevention, 17, 3116-3122. http://dx.doi.org/10.1158/1055-9965.EPI-08-0527

[14] Ghorbani, A., Moradi, A., Gookizadeh, A., Jokar, S. and Sonbolestan, S.A. (2015) Evaluation of Relationship between Breast Cancer and Migraine. Advanced Biomedical Research, 4, 14. http://dx.doi.org/10.4103/2277-9175.148297

[15] Winter, A.C., Rexrode, K.M., Lee, I.M., Buring, J.E., Tamimi, R.M. and Kurth, T. (2013) Migraine and Subsequent Risk of Breast Cancer: A Prospective Cohort Study. Cancer Causes \& Control, 24, 81-89. http://dx.doi.org/10.1007/s10552-012-0092-X

[16] Phipps, A.I., Anderson, G.L., Cochrane, B.B., Li, C.I., Wactawski-Wende, J., Ho, G.Y., et al. (2012) Migraine History, Nonsteroidal Anti-Inflammatory Drug Use, and Risk of Postmenopausal Endometrial Cancer. Hormones and Cancer, 3 , 240-248.

[17] von Elm, E., Altman, D.G., Egger, M., Pocock, S.J., Gøtzsche, P.C., Vandenbroucke, J.P., et al. (2007) Strengthening the Reporting of Observational Studies in Epidemiology (STROBE) Statement: Guidelines for Reporting Observational Studies. British Medical Journal, 335, 806-808. http://dx.doi.org/10.1136/bmj.39335.541782.AD

[18] Borenstein, M., Hedges, L.V., Higgins, J.P.T. and Rothstein, H.R. (2009) Introduction to Meta-Analysis. John Wiley \& Sons, Hoboken. http://dx.doi.org/10.1002/9780470743386

[19] Egger, M., Davey, S.G., Schneider, M. and Minder, C. (1997) Bias in Meta-Analysis Detected by a Simple, Graphical 
Test. British Medical Journal, 315, 629-634. http://dx.doi.org/10.1136/bmj.315.7109.629

[20] Liberati, A., Altman, D.G., Tetzlaff, J., Mulrow, C., Gøtzsche, P.C., Ioannidis, J.P., et al. (2009) The PRISMA Statement for Reporting Systematic Reviews and Meta-Analyses of Studies that Evaluate Healthcare Interventions: Explanation and Elaboration. British Medical Journal, 339, b2700. http://dx.doi.org/10.1136/bmj.b2700

[21] Brandes, J.L. (2006) The Influence of Estrogen on Migraine: A Systematic Review. The Journal of the American Medical Association, 295, 1824-1830. http://dx.doi.org/10.1001/jama.295.15.1824

[22] Suzuki, R., Orsini, N., Saji, S., Key, T.J. and Wolk, A. (2009) Body Weight and Incidence of Breast Cancer Defined by Estrogen and Progesterone Receptor Status-A Meta-Analysis. International Journal of Cancer, 124, 698-712. http://dx.doi.org/10.1002/ijc.23943

[23] Vrieling, A., Buck, K., Kaaks, R. and Chang-Claude, J. (2010) Adult Weight Gain in Relation to Breast Cancer Risk by Estrogen and Progesterone Receptor Status: A Meta-Analysis. Breast Cancer Research and Treatment, 123, 641649. http://dx.doi.org/10.1007/s10549-010-1116-4

[24] Althuis, M.D., Fergenbaum, J.H., Garcia-Closas, M., Brinton, L.A., Madigan, M.P. and Sherman, M.E. (2004) Etiology of Hormone Receptor-Defined Breast Cancer: A Systematic Review of the Literature. Cancer Epidemiology, Biomarkers \& Prevention, 13, 1558-1568.

[25] Phipps, A.I., Malone, K.E., Porter, P.L., Daling, J.R. and Li, C.I. (2008) Body Size and Risk of Luminal, HER2Overexpressing, and Triple-Negative Breast Cancer in Postmenopausal Women. Cancer Epidemiology, Biomarkers \& Prevention, 17, 2078-2086. http://dx.doi.org/10.1158/1055-9965.EPI-08-0206

[26] Munsell, M.F., Sprague, B.L., Berry, D.A., Chisholm, G. and Trentham-Dietz, A. (2014) Body Mass Index and Breast Cancer Risk According to Postmenopausal Estrogen-Progestin Use and Hormone Receptor Status. Epidemiologic Reviews, 36, 114-136. http://dx.doi.org/10.1093/epirev/mxt010

[27] Rasmussen, B.K. (1995) Epidemiology of Migraine. Biomedicine \& Pharmacotherapy, 49, 452-455. http://dx.doi.org/10.1016/0753-3322(96)82689-8 